\title{
The distance of the SNR Kes 75 and PWN PSR J1846-0258 system ${ }^{\star}$
}

\author{
D. A. Leahy ${ }^{1}$ and W. W. Tian ${ }^{1,2}$ \\ 1 Department of Physics \& Astronomy, University of Calgary, Calgary, Alberta T2N 1N4, Canada \\ e-mail: tww@iras.ucalgary.ca \\ 2 National Astronomical Observatories, CAS, Beijing 100012, PR China \\ Received 26 November 2007 / Accepted 24 January 2008
}

ABSTRACT

\begin{abstract}
The supernova remnant (SNR) Kes 75/PSR J1846-0258 association can be regarded as a certainty due to the accurate location of young PSR J1846-0258 at the center of Kes 75 and the detected bright radio/X-ray synchrotron nebula surrounding the pulsar. We provide a new distance estimate to the $\mathrm{SNR} /$ pulsar system by analyzing the $\mathrm{HI}$ and ${ }^{13} \mathrm{CO}$ maps, the $\mathrm{HI}$ emission and absorption spectra, and the ${ }^{13} \mathrm{CO}$ emission spectrum of Kes 75 . That there are no absorption features at negative velocities strongly argues against the widely-used large distance of 19 to $21 \mathrm{kpc}$ for Kes 75, and shows that Kes 75 is within the Solar circle, i.e. a distance $d<$ $13.2 \mathrm{kpc}$. Kes 75 is likely at a distance of 5.1 to $7.5 \mathrm{kpc}$ because the highest $\mathrm{HI}$ absorption velocity is at $95 \mathrm{~km} \mathrm{~s}^{-1}$, and no absorption is associated with a nearby HI emission peak at $102 \mathrm{~km} \mathrm{~s}^{-1}$ in the direction of Kes 75. This distance to Kes 75 gives a reasonable luminosity of PSR J1846-0258 and its PWN and also leads to a much smaller radius for Kes 75, so the age of the SNR is consistent with the spin-down age of PSR J1846-0258, confirming this pulsar as the second-youngest in the Galaxy.

Key words. supernovae: individual: Kes 75 - pulsars: individual: PSR J1846-0258 - radio lines: general - ISM: molecules -

ISM: atoms
\end{abstract}

\section{Introduction}

The supernova remnant (SNR) Kes 75 (G29.7-0.3) is one of a few examples of a shell-type remnant harboring an extremely young pulsar that powers an extended radio/X-ray synchrotron nebula. Ever since the young energetic X-ray pulsar, PSR J1846-0258, was discovered by Gotthelf et al. (2000) using the Rossi X-Ray Timing Explorer, many observations of this system at different wave-bands have been executed (Sugizaki et al. 2001; Mereghetti et al. 2002; Bock \& Gaensler 2005; Djannati-Ataï 2007; McBride et al. 2008). These observations strongly support the $\mathrm{SNR} /$ pulsar association because of both the position of PSR J1846-0258 at the geometrical centers of the SNR shell and the bright radio/X-ray nebula within the SNR.

A young pulsar usually favors a small radius of the associated SNR, but Kes 75 had an estimated radius of $10 \mathrm{pc}$ based on the previously estimated $19 \mathrm{kpc}$ distance. Caswell et al. (1975) employed the Parkes HI-line interferometer to find the most distant reliable absorption feature at velocity of $\sim 90 \mathrm{~km} \mathrm{~s}^{-1}$ in the direction of Kes 75, and suggested the lower-limit distance of $6.6 \mathrm{kpc}$ (assuming Solar-Galactic center distance $R_{0}=10 \mathrm{kpc}$ ). This drops to $5.0 \mathrm{kpc}$ using a recent measurement of $R_{0}=7.6 \pm$ $0.3 \mathrm{kpc}$ (Eisenhauer et al. 2005). Subsequent distance estimates of 19 to $21 \mathrm{kpc}$ for Kes 75 were obtained. Becker \& Helfand (1984) suggested $21 \mathrm{kpc}$ using VLA (D array) HI absorption spectrum observations with features at negative velocity. Based on $d=19$ to $21 \mathrm{kpc}$, the extremely high X-ray luminosities for Kes $75\left(\sim 4 \times 10^{37} \mathrm{erg} \mathrm{s}^{-1}\right)$, the pulsar, and the PWN require a highly implausible explosion energy $\left(\sim 10^{53} \mathrm{erg}\right)$, leading to several progenitor evolutionary scenarios (Morton et al. 2007; Helfand et al. 2003). The most likely one of the scenarios points

^ DAL and WWT gave equal contribution to the paper. to a large error in the distance estimate of $\sim 19 \mathrm{kpc}$ (Morton et al. 2007).

The $21 \mathrm{~cm}$ neutral hydrogen absorption measurements to the Galactic objects can generally provide a distance estimate with an uncertainty of $<1 \mathrm{kpc}$. Recently we used $\mathrm{HI}+{ }^{13} \mathrm{CO}$ observations to measure and revise distances to a few SNRs with high precision (Leahy \& Tian 2008; Tian et al. 2007; Tian \& Leahy 2008). In this paper, we significantly revise the distance to Kes 75 using these methods. The radio data come from $1420 \mathrm{MHz}$ continuum plus HI-line observations of the VLA Galactic Plane Survey (VGPS) and the ${ }^{13} \mathrm{CO}-$ line $(J=1-0)$ observations of the Galactic ring survey (Stil et al. 2006; Jackson et al. 2006).

\section{Analysis and results}

In Fig. 1, the VGPS $1420 \mathrm{MHz}$ continuum and one-channel $\mathrm{HI}$ images of Kes 75 are given in Galactic coordinates, showing the two emission peaks from the SNR shell. The shell is shown at higher resolution using VLA in B, C, and D array by Helfand et al. (2006). Figure 2 shows two HI-channel maps, one CO-channel map, and the $\mathrm{HI}$ absorption/emission and CO emission spectra. The HI spectra do not show any absorption at negative velocities, so Kes 75 must lie within the Solar circle, which is at $d \simeq 13.2 \mathrm{kpc}$ at $l=29.7^{\circ}$. This result significantly revises the previously widely-used value of 19 to $21 \mathrm{kpc}$ for Kes 75 . Here we use a circular rotation model with $R_{0}=7.6 \mathrm{kpc}$ and constant circular velocity $V_{0}=220 \mathrm{~km} \mathrm{~s}^{-1}$ (1985 IAU standard value), yielding a tangent point velocity at $l=29.7^{\circ}$ of $111 \mathrm{~km} \mathrm{~s}^{-1}$. This is consistent with the observation value in the direction of Kes 75 of $\sim 110 \mathrm{~km} \mathrm{~s}^{-1}$ from Fig. 2. We have also examined the HI channel images in the negative velocity range where Becker \& Helfand (1984) find the absorption features, and found no evidence of absorption. 

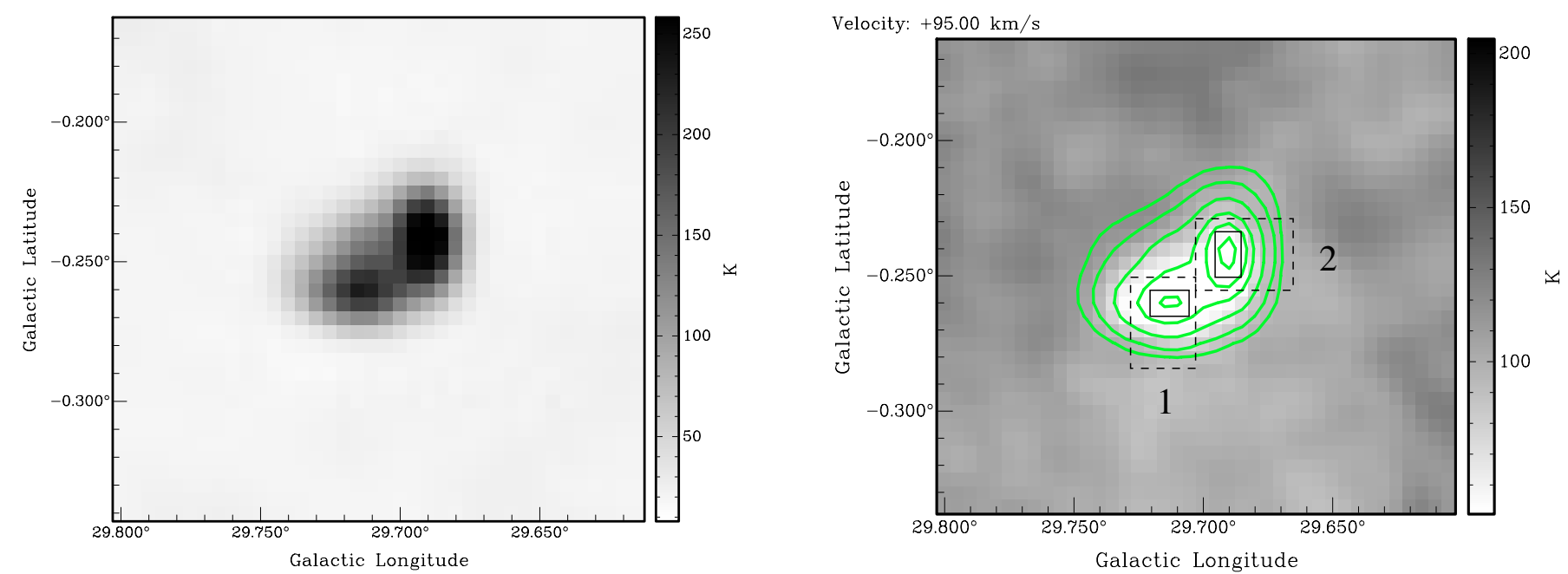

Fig. 1. The $1420 \mathrm{MHz}$ continuum image (left) and HI image (right) of Kes 75 from a single channel. The HI map has superimposed contours (28, $40,60,100 \mathrm{~K}$ ) of the $1420 \mathrm{MHz}$ continuum emission to show the SNR.

Further information on the distance of Kes 75 can be obtained by examining the absorption spectra and $\mathrm{HI}$ and $\mathrm{CO}$ channel images. At $95 \mathrm{~km} \mathrm{~s}^{-1}$ there is clear absorption seen in the HI channel map (Fig. 1, right panel) and in the absorption spectra of both continuum peaks (boxes 1 and 2 in Fig. 2) of Kes 75. To verify our method of using annulus background, we calculated an HI absorption spectrum of box 1 using an alternate background (see Fig. 2 lower left panel) and obtain almost identical spectra. Ninety-five $\mathrm{km} \mathrm{s}^{-1}$ is the highest velocity of prominent $\mathrm{HI}$ absorption appearing in the spectra (or channel images) of Kes 75, and it corresponds to a minimum distance of $5.1 \mathrm{kpc}$. Also, there is no absorption in the HI channel map (Fig. 2 top right panel) or in the $\mathrm{HI}$ absorption spectra for the $\mathrm{HI}$ emission peak at $102 \mathrm{~km} \mathrm{~s}^{-1}$ in the direction of Kes 75. Thus Kes 75 is nearer than this emission peak, which could be at either the near side or the far side of the tangent point. If this gas is on the near side, the upper limit for the distance of Kes 75 is $5.5 \mathrm{kpc}$; in the case that $\mathrm{HI}$ is on the far side, the upper limit is $7.5 \mathrm{kpc}$. We used the latter in the absence of evidence that this emission peak is on the near side of the tangent point. The $\mathrm{CO}$ emission spectra in the direction of boxes 1 and 2 both show a high brightnesstemperature cloud component at a velocity of $102 \mathrm{~km} \mathrm{~s}^{-1}$. Since there is no strong respective $\mathrm{HI}$ absorption in the spectra of Kes 75, the CO cloud should be behind Kes 75 and is probably associated with the HI-emission peak at $102 \mathrm{~km} \mathrm{~s}^{-1}$. Thus both $\mathrm{HI}$ and $\mathrm{CO}$ observations show that Kes 75 is nearer than the interstellar gas at $102 \mathrm{~km} \mathrm{~s}^{-1}$ and confirm an upper distance limit of $7.5 \mathrm{kpc}$.

There is one puzzling feature that seems to be caused by true absorption at $\sim 112 \mathrm{~km} \mathrm{~s}^{-1}$ in the direction of box 1 , but not in the direction of box 2 (Fig. 2, HI channel map, HI and CO spectra). This cannot be due to diffuse HI, or else it would appear in absorption against both continuum peaks of Kes 75. This feature is probably caused by a compact HI cloud at $112 \mathrm{~km} \mathrm{~s}^{-1}$ velocity. Since the cloud is compact, it could either be at the tangent point or it could be moving at a peculiar velocity, and elsewhere along the line-of-sight. If the cloud has no peculiar velocity it provides a lower limit to the distance to Kes 75 of the tangent point distance $(6.6 \mathrm{kpc})$ and also confirms that the $\mathrm{HI}$ at $102 \mathrm{~km} \mathrm{~s}^{-1}$ is on the far side of the tangent point. If it does have a peculiar velocity, then this cloud gives no useful distance information.
In summary, Kes 75 should be in the distance range of 5.1 to $7.5 \mathrm{kpc}$ because of the absorption at $95 \mathrm{~km} \mathrm{~s}^{-1}$ and absence of absorption at $102 \mathrm{~km} \mathrm{~s}^{-1}$.

\section{Discussion}

We find strong evidence about the distance to Kes 75, and if disagrees with what is given by Becker \& Helfand (1984). By testing different background regions, we were not able to obtain similar HI absorption spectra to those shown in that study. The VGPS data used here is superior to their data in sensitivity, and it also includes single-dish observations from the $100 \mathrm{~m}$ Green Bank telescope, yielding the large-scale HI and continuum emission in addition to small-scale emission. This is probably one reason we get a different, better, result.

Next we discuss implications of the shorter distance to Kes 75. The new distance gives a much smaller radius for Kes 75: the radio and X-ray sizes nearly match (Morton et al. 2007 ) and give a radius of $1.60 \mathrm{arcmin}$. Our new distance range gives a physical radius range of 2.37 to $3.49 \mathrm{pc}$. We write the distance to Kes 75 as $d=d_{6} \times 6 \mathrm{kpc}$, so the radius is $2.80 d_{6} \mathrm{pc}$. As estimate of the age of the SNR, we use the spin-down age of 723 years for PSR J1846-0258 from Gotthelf et al. (2000). Livingstone et al. (2006) use measurements of braking index of PSR J1846-0258 to put an upper limit on age of 884 yr, confirming the spin-down age estimate. Assuming free expansion, the derived expansion velocity is then $3800 d_{6} \mathrm{~km} \mathrm{~s}^{-1}$ with this spindown age and a radius of $2.80 d_{6}$ pc. If the SNR is expanding according to Sedov evolution, the current Sedov shock velocity is 0.4 times the average expansion velocity, giving a shock velocity of $1500 d_{6} \mathrm{~km} \mathrm{~s}^{-1}$. For the more realistic case, the initial phases will be dominated by free-expansion, so the current shock velocity will be higher than the Sedov estimate and lower than the free expansion estimate. However, we note that Helfand et al. (2006) estimate the expansion velocity of Kes 75 using the Si X-ray line width to obtain $\sim 3700 \mathrm{~km} \mathrm{~s}^{-1}$. This indicates that Kes 75 is probably closer to the free-expansion phase.

The electron temperature of the high-temperature component in the X-ray spectrum is $1.4-1.5 \mathrm{keV}$ (Morton et al. 2007), which corresponds to a shock velocity (for electron-ion equilibration) of $1200 \mathrm{~km} \mathrm{~s}^{-1}$. Since the minimum shock velocity 

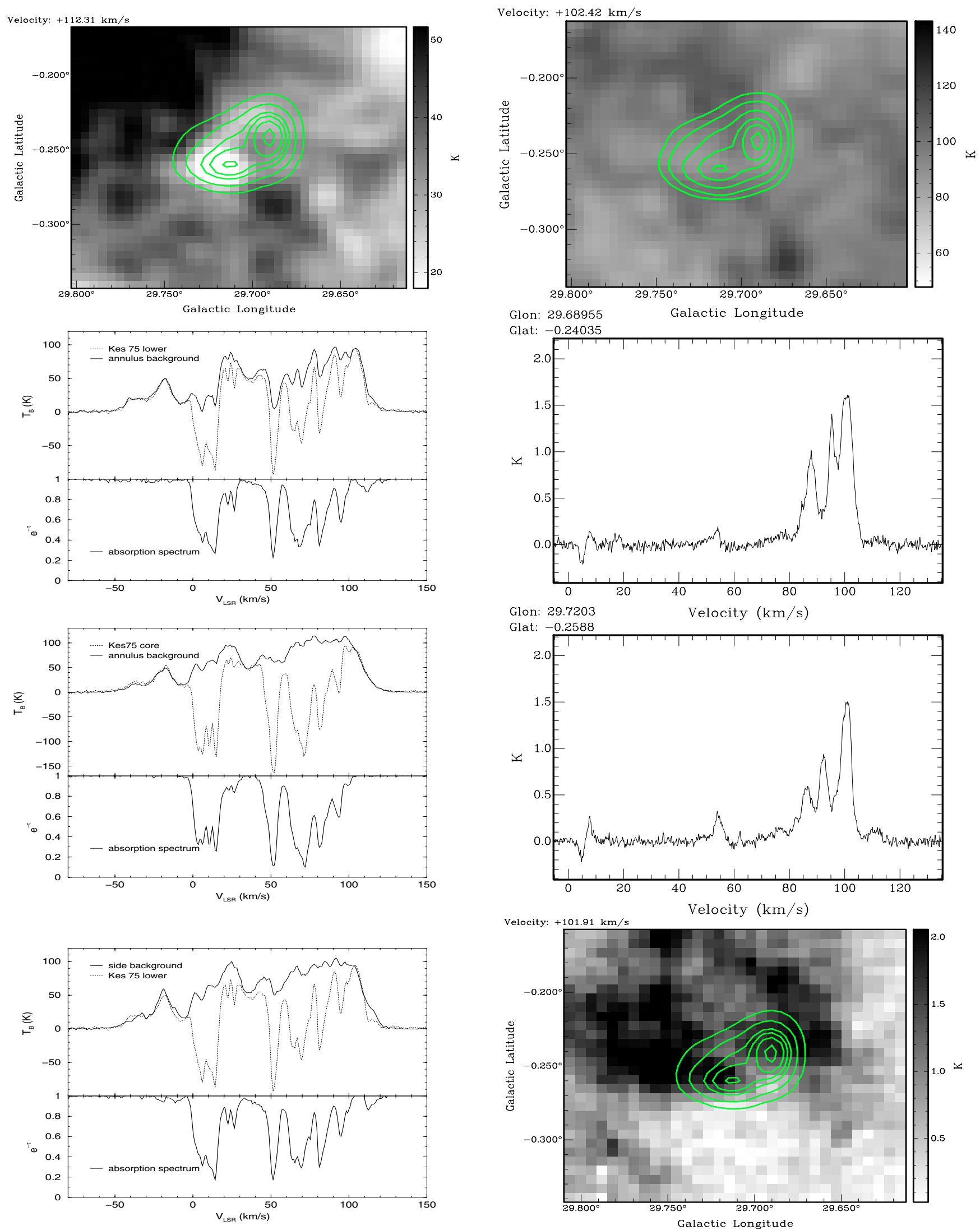

Fig. 2. Two channel HI images of Kes 75 (top row). The second and third rows show the HI spectra (left) and the CO emission spectra (right), extracted from boxes 1 and 2 shown in Fig. 1. The bottom row shows an additional HI spectra (left) with the source spectrum from box 1 and background spectrum from the area at $l[29.705,29.725], b[-0.285,-0.310]$, and one-channel CO image (right). Both the HI and CO maps have superimposed the same contours as Fig. 1. 
is $1500 d_{6} \mathrm{~km} \mathrm{~s}^{-1}$, there must not be full electron-ion equilibration: one finds a maximum electron-to-ion temperature ratio of 0.64 . The shock velocity derived from the electron temperature increases in this case. For an electron-to-ion temperature ratio of 0.1 , one obtains a shock velocity of $3800 \mathrm{~km} \mathrm{~s}^{-1}$. Thus the electron-to-on temperature ratio is restricted to the range of 0.10 to 0.64 for Kes 75 .

Morton et al. (2007) give X-ray emitting gas masses in hot $(1.4 \mathrm{keV})$ and cool $(0.23 \mathrm{keV})$ components. For our new distance, the masses are reduced to reasonable values: $5.3 f_{1}^{1 / 2} d_{6}^{5 / 2} M_{\odot}$ for the cool component and $0.84 f_{1}^{1 / 2} d_{6}^{5 / 2} M_{\odot}$ for the hot component with $f_{1}$ the filling factor. They interpret the cool component as shocked interstellar medium (ISM) or circumstellar material (CSM) and the hot component as reverse-shocked ejecta. That two temperature components are seen is clear evidence that the ejecta has not fully entered the Sedov phase. The shocked-ejecta mass is likely to be smaller than the ISM/CSM shocked mass since only a small part of the ejecta has been shocked so far. The expansion should have already slowed down somewhat from the initial ejection velocity, so the current shock velocity and electron-to-ion temperature ratio should be somewhere between the Sedov estimates $\left(1500 d_{6} \mathrm{~km} \mathrm{~s}^{-1}\right.$ and 0.64$)$ and the free-expansion values $\left(3800 d_{6} \mathrm{~km} \mathrm{~s}^{-1}\right.$ and 0.10$)$.

Using the Sedov model and age of $723 \mathrm{yr}$, the observed outer shock radius yields an estimate of the explosion energy $\left(E_{0}\right)$ divided by interstellar density $\left(n_{0}\right): E_{0} / n_{0}=0.69 d_{6}^{5} \times 10^{50} \mathrm{erg}$. The X-ray spectral fitting yields an estimate of the density for the shocked CSM/ISM component of $n=100 f_{1}^{-1 / 2} d_{6}^{-1 / 2} \mathrm{~cm}^{-3}$ and $n=64 f_{1}^{-1 / 2} d_{6}^{-1 / 2} \mathrm{~cm}^{-3}$ for the SE and SW limbs of Kes 75, respectively. From the inferred X-ray post-shock density $(n)$ and using a compression rate of 3 (from the observed radio spectral index of 0.7, see Droge et al. 1987), we obtain a preshock density $n_{0}=n / 3 \simeq 27 f_{1}^{-1 / 2} d_{6}^{-1 / 2} \mathrm{~cm}^{-3}$ and an explosion energy of $E_{0}=1.9 d_{6}^{9 / 2} f_{1}^{-1 / 2} \times 10^{51} \mathrm{erg}$. This is entirely consistent with what is expected for a core-collapse explosion now, contrary to the problems caused by having a large distance to Kes 75 .

For PSR J1846-0258, the pulsar-plus-nebular luminosity in the $3-10 \mathrm{keV}$ band is $7.8 \times 10^{34} d_{6}^{2} \mathrm{erg} \mathrm{s}^{-1}$, so the pulsar-plusnebula luminosity to spin-down luminosity has a slightly lower ratio $\left(0.6 d_{6}^{2}\right)$ than for the Crab, rather than the previously inferred, much higher ratio.

In summary, we find observational evidence that the distance to Kes 75 is about $6 \mathrm{kpc}$ and that it has a normal explosion energy and harbors a Crab-like rotation powered pulsar and pulsar wind nebula. This is unusual in that the pulsar has a high value for magnetic field $\left(5 \times 10^{13}\right.$ Gauss), and it is very young. It also exploded in a somewhat high-density environment: $n_{0} \simeq 27 \mathrm{~cm}^{-3}$.

We can estimate the absorbing column density to Kes 75 $N_{\mathrm{HI}}$ from the $\mathrm{HI}$ absorption spectrum (Fig. 2) by $N_{\mathrm{HI}}=1.9 \times$ $10^{18} \tau \Delta v T_{\mathrm{s}} \mathrm{cm}^{-2}$ (Dickey \& Lockman 1990). Assuming HI spin temperature $T_{\mathrm{s}}$ of $50 \mathrm{~K}$, we sum over the absorption features to obtain $N_{\mathrm{HI}}$ of $\sim 3 \times 10^{22}$ and $\sim 4 \times 10^{22} \mathrm{~cm}^{-2}$ in front of regions 1 and 2, respectively. These values agree with that of $N_{\mathrm{H}}=4 \times 10^{22} \mathrm{~cm}^{-2}$ derived from X-ray observations to Kes 75 . Given the high column density to Kes 75 , the derived extinction is $A_{\mathrm{V}} \sim 21 \mathrm{mag}$, which would explain why no historical SN has been observed associated with Kes 75 around the time of explosion in 1300 A.D.

Acknowledgements. D.A.L. and W.W.T. acknowledge support from the Natural Sciences and Engineering Research Council of Canada. W.W.T. acknowledges support from the Natural Science Foundation of China. This publication makes use of molecular line data from the Boston University-FCRAO Galactic Ring Survey. The NRAO is a facility of the National Science Foundation operated under cooperative agreement by Associated Universities, Inc.

\section{References}

Becker, R. H., \& Helfand, D. J. 1984, ApJ, 283, 154

Bock, D. C. J., \& Gaensler, B. M. 2005, ApJ, 626, 343

Caswell, J. L., Murray, J. D., \& Roger, R. S. 1975, A\&A, 45, 239

Dickey, J. M., \& Lockman, F. J. 1990, ARA\&A, 28, 215

Djannati-Atai,, A. for the HESS Collaboration, 2007, in 30th ICRC, Merida, Mexico [arXiv: 0710.2247]

Droge, W., Lerche, I., \& Shlickeiser, R. 1987, A\&A, 178, 252

Eisenhauer, F., Genzel, R., Alexander, T., et al. 2005, ApJ, 628, 246

Gotthelf, E. V., Vasisht, G., Boylan-Kolchin, M., \& Torii, K. 2000, ApJL, 542, 37

Helfand, D. J., Collins, B. F., \& Gotthelf, E. V. 2003, ApJ, 582, 783

Helfand, D. J., Becker, R. H., White, R. L., Fallon, A., \& Tuttle, S. 2006, AJ, 131,2525

Jackson, J. M., Rathborne, J. M., Shah, R. H., et al. 2006, ApJS, 163, 145

Leahy, D. A., \& Tian, W. W. 2008, AJ, 135, 167

Livingstone, M. A., Kaspi, V. M., Gotthelf, E. V., \& Kuiper, L. 2006, ApJ, 647, 1286

McBride, V. A., Dean, A. J., \& Bazzano, A. 2008, A\&A, 477, 249

Mereghetti, S., Bandiera, R., Bocchino, F., \& Israel, G. L. 2002, ApJ, 574, 873

Morton, T. D., Slane, P., \& Borkowski, K. J. 2007, ApJ, 667, 219

Stil, J. M., Taylor, A. R., Dickey, J. M., et al. 2006, AJ, 132, 1158

Sugizaki, M., Mitsuda, K., Kaneda, H., et al. 2001, ApJS, 134, 77

Tian, W. W., \& Leahy, D. A. 2008, ApJ, dol:10.1086/529120

Tian, W. W., Leahy, D. A., \& Wang, Q. D. 2007, A\&A, 474, 541 